\title{
ARBITRARY ORDER PERTURBATION EXPANSION APPLIED TO STEADY-STATE NONLINEAR KERR EFFECT RELAXATIONS IN COUPLED AC AND DC WEAK FIELDS
}

\author{
M.N. IIOUNKONNOU* \\ Laboratoire de Modélisation de Systèmes Physiques \\ Physics Department, Faculté des Sciences et Techniques \\ Université Nationale du Benin \\ B.P. 526-Cotonou, Benin \\ AND A. Ronveaux \\ Laboratoire de Pliysique Mathématique \\ Facultés Universilaires Notre-Dame de la Paix \\ Rue de Bruxclles, 61, 5000 Namur, Belgium
}

(Received June 11, 1992)

The steady state response of the nonlinear Kerr effect to a small ac field superimposed on a dc ficld is analysed using second-order perturbation theory. We justify the truncature effect entailed in solving the infinite set of differential equations that yield the electric polarization and birefringence, and hence draw inferences from the appearance of single and double harmonics as the fundamental frequencies of nonlinear Kerr effect. The addition of small inertial effects in the solution of this phenomenon results in the appearance of a contribution within the master matrix which is proportional to the square of the frequency corresponding to the order of perturbation. The study is based on the Smoluchowski equation of rotational Brownian motion.

PACS numbers: $33.55 . \mathrm{Fi}, 05.40 .+\mathrm{j}$

*Present address: Physics Department, Faculté des Sciences, Université Libre de Bruxelles, Campus de la Plaine, CP 223, Boulevard du Triomphe, 1050-Bruxelles, Belgium. 


\section{Introduction}

When an initially isotropic molecular fluid is subject to an external electric field, the fluid molecules involved are oriented to and, in some instances, deformed by the action of the field on the permanent and induced dipole moments of the molecules. The fluid becomes birefringent. This phenomenon is called the electrooptical birefringence or the Kerr effect. For a weak field, the degree of birefringence is proportional to the square of the field strength. The proportionality constant multiplied by an appropriate constant factor defines the Kerr constant..

In order to interpret such physical processes, it is necessary to describe the orientation motion of a molecule of a liquid. In a dense fluid, a molecule will experience many collisions as it rotates from position to position. This view of the dynamics of molecular liquids suggests that the process is diffusion-like in nature.

Although a molecule in a fluid rotates along a deterministic trajectory according to Euler's equations of motion, this motion is sufficiently complex so that it may be viewed as proceeding through a rather randomly chosen path. Since the orientational motion of a molecule of the liquid is affected by collisions and has a similarly complex trajectory, it is possible to develop a diffusion model for orientational dynamics.

Langevin [1] proposed the first mathematical theory of Kerr electric birefringence (KEB), based on the orientation of anisotropic molecules due to the introduction of an orientation function of molecules possessing a symmetry axis. Born [2] generalized this theory by introducing the permanent dipole moment. All present theories involve these basic ideas.

The Kerr electrooptic phenomenon was studied intensively in a continuous regime, often in a pure alternating regime, and by Pauthenier [3] in an impulse regime. In the last two decades, the Laboratoire de Physique Appliquée de l'Université de Perpignan, has contributed much to a better understanding of these two regimes [4-8]. Filippini [9] measured experimentally the Kerr dispersion constant when an alternating field superimposed on a unidirectional field is applied to the liquid. Theoretical studies of Coffey and Paranjape [10], Morita [11], Morita and Watanabe [12] and those of Alexiewicz [13] on the dielectric relaxation and KEB in alternating and unidirectional fields have already appeared.

Peterlin and Stuart [14] obtained solutions of the electric birefringence in a sinusoidal electric field $E(t)=E_{0} \cos (\omega t)$ for the cases of pure induced dipole and pure permanent dipole orientations. The solutions are limited to infinitely small fields. The general case of induced and permanent dipoles coexisting on the particle was first solved by Ogawa and Oka [15] for a very low sinusoidal electric field $E(t)=E_{0} \sin (\omega t)$. Later the same problem was treated by Thurston and Bowling [16] for a sinusoidal electric field $E(t)=E_{0} \cos (\omega t)$.

Most of these works were made in the linear regime.

In a previous paper [17] we extended the already existent theory of linear Kerr effect, to treat the nonlinear regime in superimposed ac and dc fields for the general case of molecules with permanent and induced dipole moments. We adopted a perturbation approach to treat the general nonlinear regime - within the classical framework of the Smoluchowski theory - and proposed steady-state 
analytical expressions for nonlinear electric polarization and birefringence in terms of KEB fundamental harmonics and characteristic eigenvectors obtained from a vector representation of the main system of equations.

In the present study, we pursue the discussions mentioned above in two directions. First, we analyse, using the same perturbation theory, the truncature effect arising from the reduction of the main infinite sets of equations to two, three, or four sets of differential equations in order to describe the nonlinear Kerr effect relaxations. We deal with steady-state analytical expressions for nonlinear electric polarization and birefringence in terms of KEB fundamental harmonics and characteristic eigenvectors obtained from a vector representation of the main system of equations. Second, we generalize this perturbation approach - within the framework of the Sack formalism - in order to describe the influence of small inertial effects on the nonlinear electric polarization and birefringence for a sudden application of both ac and dc fields. We also obtain the appropriate steady-state solutions in terms of KEB fundamental harmonics and characteristic eigenvectors. These expressions reveal the appearance of a contribution within a master matrix which is proportional to the square of the frequency corresponding to the order of perturbation.

In Sec. 2 we present a brief theoretical introduction, with the main sets of differential equations, usually truncated to two sets of equations in order to describe the nonlinear electric polarization and birefringence. Next, we develop the perturbation expansion and justify the degree of truncature necessary to obtain the essential molecular information (Sec. 3). In the last section, we extend this perturbation theory to include small inertial effects on this Kerr physical process in any order of perturbation.

\section{General theory}

Upon the assumption that the particle is axially symmetric and non-interacting with each other, the orientational movement of the molecule may be described by the rotational Smoluchowski diffusion equation for the angular distribution function $f=f(\theta, t)$ :

$$
D^{-1} \frac{\partial f}{\partial t}=\frac{1}{\sin \theta} \frac{\partial}{\partial \theta}\left[\sin \theta\left(\frac{\partial f}{\partial \theta}+\frac{1}{k T} \frac{\partial W}{\partial \theta} f\right)\right]
$$

where $D$ is the rotational diffusion constant around the transverse axis of the molecule, $k T$ is the thermal energy, $\theta$ is the angle between the symmetry axis and the field direction, $t$ is the time, and $W$ - the orientational energy of the molecule - is given by

$$
W=-\mu E(t) \cos \theta-\frac{1}{2}\left(\alpha_{1}-\alpha_{2}\right) E^{2}(t) \cos ^{2} \theta
$$

where $\mu$ is the permanent dipole moment along the axis of symmetry of the molecule; $\Delta \alpha=\alpha_{1}-\alpha_{2}$, the diference of the molccular polarizability between parallel $\alpha_{1}$ and perpendicular $\alpha_{2}$ components to the molecular axis. $E(t)$ is the external electric field. $\alpha_{1}$ and $\alpha_{2}$ may gencrally be functions of the electric field and can be expressed in a power series of $E$ as follows [18]:

$$
\alpha_{i}=a_{i}+b_{i} E^{2}+c_{i} E^{4}+\ldots \quad(i=1,2) .
$$


In the following, however, we regard the polarizability as a constant by neglecting effects due to the hyperpolarizability.

Equation (1) may be solved by expanding the distribution function $f=$ $f(\theta, \imath)$ in the Legendre polynomials $[5-8,11-13,17,19-22]$ :

$$
f(\theta, t)=\sum_{n=0}^{\infty} C_{n}(t) P_{n}(\cos \theta),
$$

where $C_{n}(t)$ is a function of time and $P_{n}$ is the Legendre polynomial of degree $n$,

$$
C_{n}(t)=\frac{2 n+1}{2} \int_{-1}^{+1} f(\theta, l) P_{n}(\cos \theta) \mathrm{d}(\cos \theta) \text {. }
$$

Substituting $f$ into Eq. (1) and using the recursion relations between the Legendre polynomials, one can simply obtain the recurrence relation as follows $[5-8,11-13,17,19-22]$ :

$$
\begin{aligned}
D^{-1} \frac{\mathrm{d}}{\mathrm{dt}}\langle & \left.P_{n}(u)\right\rangle(t)=-n(n+1)\left\langle P_{n}(u)\right\rangle(t) \\
& +\gamma \frac{n(n+1)}{2 n+1}\left[\left\langle P_{n-1}(u)\right\rangle(l)-\left\langle P_{n+1}(u)\right\rangle(t)\right] \\
& +\beta \frac{n(n+1)}{2 n+1}\left[\frac{2 n+1}{(2 n-1)(2 n+3)}\left\langle P_{n}(u)\right\rangle(t)\right. \\
& \left.+\frac{n-1}{2 n-1}\left\langle P_{n-2}(u)\right\rangle(l)-\frac{n+2}{2 n+3}\left\langle P_{n+2}(u)\right\rangle(t)\right],
\end{aligned}
$$

where

$$
\begin{aligned}
& \left\langle P_{0}(u)\right\rangle(t)=1, \quad \gamma=\gamma(t)=\frac{\mu}{k T} E(t) \quad u=\cos \theta, \quad \beta=\beta(t)=\frac{\Delta \alpha}{k T} E^{2}(t), \\
& \left\langle P_{n}(u)\right\rangle(t) \equiv \int_{-1}^{+1} f(u, t) P_{n}(u) \mathrm{d} u \equiv y_{n}(l),
\end{aligned}
$$

for the normalized distribution funclion $\int_{-1}^{+1} f(u, t) \mathrm{d} u=1$, in which angular brackets represent the ensemble averagc and $f(u, l)$ is the normalized distribution function in the new variable $u$.

The electric polarization and birefringence are directly connected to $y_{1}(t)$ and $y_{2}(t)[6,12,17,22]$. But the precision of the calculation of these quantities depends on the degree of the truncature solving Eq. (6). In the previous paper, we limited to $n=2$ by taking up the terms of second power of $E(t)$ and fourth power of $E(t)$ for nonlinear electric polarization and electric birefringence, respectively

$$
\begin{aligned}
& D^{-1} \frac{\mathrm{d} y_{1}(t)}{\mathrm{d} t}=-\left(2-\frac{2 \beta}{5}\right) y_{1}(t)-\frac{2 \gamma}{3} y_{2}(t)+\frac{2 \gamma}{3}, \\
& D^{-1} \frac{\mathrm{d} y_{2}(t)}{\mathrm{d} t}=\frac{6 \gamma}{5} y_{1}(l)-\left(6-\frac{2 \beta}{7}\right) y_{2}(l)+\frac{2 \beta}{5}, \\
& y_{i}(0)=0, \quad i=1,2 .
\end{aligned}
$$

Various papers were devoted to the approximation of analytical and numerical treatments of Eq. (6) or its variants for the dielectric relaxation and Kerr electric birefringence in numerous regimes of external electric fields [11-14, 19, 20-24]. 
IIounkonnou $[22,26]$ has recently used the Runge-Kutta method to solve this equation by including the nonlinear and inertial effects for a sudden application of an alternating field superimposed on an unidirectional field. He has given an exact analytical solution to the same equation, when one applies the unidirectional field [27].

Mathematically exact solutions of Eqs. (7) for an alternating field superimposed on an unidirectional field are difficult to obtain. In the previous paper, we emphasized the usefulness of exact steady-state solutions and used a second-order perturbation theory in order to solve Eqs. (7a) for

$$
E(t)=E_{\mathrm{c}}+E_{0} \cos \omega t \text {. }
$$

IIere, we adopt the same perturbation approach to obtain the steady-state solutions $y_{1}(l)$ and $y_{2}(t)$ from systems $(7 \mathrm{~b})$ and $(7 \mathrm{c})$ for electric polarization and birefringence, using a step by step truncature procedure including, respectively, $y_{3}(t)$ and $y_{4}(t)$ in system $(7 \mathrm{a})$ :

$$
\begin{aligned}
& D^{-1} \frac{\mathrm{d} y_{1}(t)}{\mathrm{d} t}=-\left(2-\frac{2 \beta}{5}\right) y_{1}(t)-\frac{2 \gamma}{3} y_{2}(t)-\frac{2 \beta}{5} y_{3}(t)+\frac{2 \gamma}{3}, \\
& D^{-1} \frac{\mathrm{d} y_{2}(t)}{\mathrm{d} t}=\frac{6 \gamma}{5} y_{1}(t)-\left(6-\frac{2 \beta}{7}\right) y_{2}(t)-\frac{6 \gamma}{5} y_{3}(t)+\frac{2 \beta}{5}, \\
& D^{-1} \frac{\mathrm{d} y_{3}(t)}{\mathrm{d} t}=\frac{24 \beta}{35} y_{1}(t)+\frac{12 \gamma}{7} y_{2}(t)-\left(12-\frac{4 \beta}{15}\right) y_{3}(t),
\end{aligned}
$$

and

$$
\begin{aligned}
& D^{-1} \frac{\mathrm{d} y_{1}(t)}{\mathrm{d} t}=-\left(2-\frac{2 \beta}{5}\right) y_{1}(t)-\frac{2 \gamma}{3} y_{2}(t)-\frac{2 \beta}{5} y_{3}(t)+\frac{2 \gamma}{3} \\
& D^{-1} \frac{\mathrm{d} y_{2}(t)}{\mathrm{d} t}=\frac{6 \gamma}{5} y_{1}(t)-\left(6-\frac{2 \beta}{7}\right) y_{2}(t)-\frac{6 \gamma}{5} y_{3}(t)-\frac{24 \beta}{35} y_{4}(t)+\frac{2 \beta}{5}, \\
& D^{-1} \frac{\mathrm{d} y_{3}(t)}{\mathrm{d} t}=\frac{24 \beta}{35} y_{1}(t)+\frac{12 \gamma}{7} y_{2}(t)-\left(12-\frac{4 \beta}{15}\right) y_{3}(t)-\frac{12 \gamma}{7} y_{4}(t), \\
& D^{-1} \frac{\mathrm{d} y_{4}(t)}{\mathrm{d} t}=\frac{20 \beta}{21} y_{2}(t)+\frac{20 \gamma}{9} y_{3}(t)-\left(20-\frac{20 \beta}{77}\right) y_{4}(t) .
\end{aligned}
$$

\section{Perturbation expansion: truncature effects}

Using the following reduced variables:

$$
t_{1}=D t, \quad \omega^{\prime}=\frac{\omega}{D}
$$

and writing

$$
\begin{aligned}
& E\left(t_{1}\right)=E_{\mathrm{c}}\left(1+\varepsilon \cos \omega^{\prime} \iota_{1}\right), \\
& \gamma\left(t_{1}\right)=\gamma_{\mathrm{c}}\left(1+\varepsilon \cos \omega^{\prime} \iota_{1}\right), \\
& \beta\left(t_{1}\right)=\beta_{\mathrm{c}}\left(1+\varepsilon \cos \omega^{\prime} \iota_{1}\right)^{2},
\end{aligned}
$$

where

$$
\varepsilon=\frac{E_{0}}{E_{\mathrm{c}}}, \quad \gamma_{\mathrm{c}}=\frac{\mu}{k T} E_{\mathrm{c}}, \quad \beta_{\mathrm{c}}=\frac{\Delta \alpha}{k T} E_{\mathrm{c}}^{2},
$$


the systems (7) may be translated into vector representation as follows:

$$
\frac{\mathrm{d} \boldsymbol{Y}\left(t_{1}, \varepsilon\right)}{\mathrm{d} t}=\left[\boldsymbol{A}\left(t_{1}, \varepsilon\right)\right] \boldsymbol{Y}\left(\iota_{1}, \varepsilon\right)+\boldsymbol{F}\left(t_{1}, \varepsilon\right), \quad \boldsymbol{Y}(0, \varepsilon)=0
$$

where:

a) For the representation of system (7a)

$$
\begin{aligned}
& {[\boldsymbol{A}]=\left(\begin{array}{ll}
-2+2 \beta / 5 & -2 \gamma / 3 \\
6 \gamma / 5 & -6+2 \beta / 7
\end{array}\right), \quad \boldsymbol{F}=\left(\begin{array}{c}
2 \beta / 3 \\
2 \beta / 5
\end{array}\right),} \\
& \boldsymbol{Y}=\left(\begin{array}{l}
y_{1} \\
y_{2}
\end{array}\right) ; \quad \frac{\mathrm{d} \boldsymbol{Y}\left(t_{1}, \varepsilon\right)}{\mathrm{d} t}=\left(\begin{array}{c}
\mathrm{d} y_{1} / \mathrm{d} t \\
\mathrm{~d} y_{2} / \mathrm{d} t
\end{array}\right)
\end{aligned}
$$

with $\beta=\beta\left(t_{1}\right), \gamma=\gamma\left(t_{1}\right) ; \varepsilon$ is a small parameter.

Particular cases of ordinary linear electric birefringence of Kerr (KEB) were treated by Morita and Watanabe [11, 12, 23] and Déjardin and Débiais [21] by considering

$$
[\boldsymbol{A}]=\left(\begin{array}{cc}
-2 & 0 \\
6 \gamma / 5 & -6+2 \beta / 7
\end{array}\right) .
$$

The same situation was examined by Schwarz [24] for purely apolar molecules $(\gamma=0)$. Okonski [25] studied the purely polar molecules $(\beta=0)$.

b) For the representation of system (7b)

$$
\begin{aligned}
{[\boldsymbol{A}] } & =\left(\begin{array}{ccc}
-2+2 \beta / 5 & -2 \gamma / 3 & -2 \beta / 5 \\
6 \gamma / 5 & -6+2 \beta / 7 & -6 \gamma / 5 \\
24 \beta / 35 & 12 \gamma / 7 & -12+4 \beta / 15
\end{array}\right), \quad \boldsymbol{F}=\left(\begin{array}{c}
2 \beta / 3 \\
2 \beta / 5 \\
0
\end{array}\right), \\
\boldsymbol{Y} & =\left(\begin{array}{l}
y_{1} \\
y_{2} \\
y_{3}
\end{array}\right) ; \quad \frac{\mathrm{d} \boldsymbol{Y}\left(\iota_{1}, \varepsilon\right)}{\mathrm{d} t}=\left(\begin{array}{c}
\mathrm{d} y_{1} / \mathrm{d} t \\
\mathrm{~d} y_{2} / \mathrm{d} \iota \\
\mathrm{d} y_{3} / \mathrm{d} t
\end{array}\right) .
\end{aligned}
$$

c) For the representation of system (7c)

$$
\begin{gathered}
{[\boldsymbol{A}]=\left(\begin{array}{cccc}
-2+2 \beta / 5 & -2 \gamma / 3 & -2 \beta / 5 & 0 \\
6 \gamma / 5 & -6+2 \beta / 7 & -6 \gamma / 5 & -24 \beta / 35 \\
24 \beta / 35 & 12 \gamma / 7 & -12+4 \beta / 15 & 12 \gamma / \tau \\
0 & 20 \beta / 21 & 20 \gamma / 9 & -20+20 \beta / 77
\end{array}\right)} \\
\boldsymbol{F}=\left(\begin{array}{c}
2 \beta / 3 \\
2 \beta / 5 \\
0 \\
0
\end{array}\right), \quad \boldsymbol{Y}=\left(\begin{array}{l}
y_{1} \\
y_{2} \\
y_{3} \\
y_{4}
\end{array}\right) ; \quad \frac{\mathrm{d} \boldsymbol{Y}\left(\iota_{1}, \varepsilon\right)}{\mathrm{d} t}=\left(\begin{array}{l}
\mathrm{d} y_{1} / \mathrm{d} t \\
\mathrm{~d} y_{2} / \mathrm{d} t \\
\mathrm{~d} y_{3} / \mathrm{d} \iota \\
\mathrm{d} y_{4} / \mathrm{d} \iota
\end{array}\right)
\end{gathered}
$$

In the following, we consider the general case of molecules having permanent and induced dipole moments without any approximations of above mentioned molecular parameters $\gamma\left(l_{1}\right)$ and $\beta\left(l_{1}\right)$. 
By expanding the dependent variables $\left[\boldsymbol{A}\left(t_{1}, \varepsilon\right)\right], \boldsymbol{Y}\left(t_{1}, \varepsilon\right)$, and $\boldsymbol{F}\left(t_{1}, \varepsilon\right)$ as a power series in $\varepsilon$, we generate a solution to any desired order in $\varepsilon$. Hence, we have

$$
\left[\boldsymbol{A}\left(t_{1}, \varepsilon\right)\right]=\left[\boldsymbol{A}_{0}\right]+\varepsilon\left[\boldsymbol{A}_{1}\right]+\varepsilon^{2}\left[\boldsymbol{A}_{2}\right]+\ldots
$$

where:

d) For system (7a)

$$
\begin{aligned}
& {\left[\boldsymbol{A}_{0}\right]=\left(\begin{array}{cc}
-2+2 \beta_{c} / 5 & -2 \gamma_{c} / 3 \\
6 \gamma_{c} / 5 & -6+2 \beta_{c} / 7
\end{array}\right),} \\
& {\left[\boldsymbol{A}_{1}\right]=\cos \omega^{\prime} t_{1}\left(\begin{array}{cc}
4 \beta_{c} / 5 & -2 \gamma_{c} / 3 \\
6 \gamma_{c} / 5 & 4 \beta_{c} / 7
\end{array}\right) \equiv \cos \omega^{\prime} t_{1}\left[a_{1}\right],} \\
& {\left[\boldsymbol{A}_{2}\right]=\cos ^{2} \omega^{\prime} t_{1}\left(\begin{array}{cc}
2 \beta_{\mathrm{c}} / 5 & 0 \\
0 & 2 \beta_{\mathrm{c}} / 7
\end{array}\right) \equiv \cos ^{2} \omega^{\prime} t_{1}\left[a_{2}\right] .}
\end{aligned}
$$

c) For system (7b)

$$
\begin{aligned}
& {\left[\boldsymbol{A}_{0}\right]=\left(\begin{array}{ccc}
-2+2 \beta_{\mathrm{c}} / 5 & -2 \gamma_{\mathrm{c}} / 3 & -2 \beta_{\mathrm{c}} / 5 \\
6 \gamma_{\mathrm{c}} / 5 & -6+2 \beta_{\mathrm{c}} / 7 & -6 \gamma_{\mathrm{c}} / 5 \\
24 \beta_{\mathrm{c}} / 35 & 12 \gamma_{\mathrm{c}} / 7 & -12+4 \beta_{\mathrm{c}} / 15
\end{array}\right)} \\
& {\left[\boldsymbol{A}_{1}\right]=\cos \omega^{\prime} \iota_{1}\left(\begin{array}{ccc}
4 \beta_{\mathrm{c}} / 5 & -2 \gamma_{\mathrm{c}} / 3 & -4 \beta_{\mathrm{c}} / 5 \\
6 \gamma_{\mathrm{c}} / 5 & 4 \beta_{\mathrm{c}} / 7 & -6 \gamma_{\mathrm{c}} / 5 \\
48 \beta_{\mathrm{c}} / 35 & 12 \gamma_{\mathrm{c}} / 7 & -8 \beta_{\mathrm{c}} / 15
\end{array}\right) \equiv \cos \omega^{\prime} t_{1}\left[\boldsymbol{a}_{1}\right]} \\
& {\left[\boldsymbol{A}_{2}\right]=\cos ^{2} \omega^{\prime} t_{1}\left(\begin{array}{ccc}
2 \beta_{\mathrm{c}} / 5 & 0 & -2 \beta_{\mathrm{c}} / 5 \\
0 & 2 \beta_{\mathrm{c}} / 7 & 0 \\
24 \beta_{\mathrm{c}} / 35 & 0 & -4 \beta_{\mathrm{c}} / 15
\end{array}\right) \equiv \cos ^{2} \omega^{\prime} t_{1}\left[\boldsymbol{a}_{2}\right] .}
\end{aligned}
$$

f) For system (7c)

$$
\begin{aligned}
& {\left[\boldsymbol{A}_{0}\right]=\left(\begin{array}{cccc}
-2+2 \beta_{\mathrm{c}} / 5 & -2 \gamma_{c} / 3 & -2 \beta_{c} / 5 & 0 \\
6 \gamma_{\mathrm{c}} / 5 & -6+2 \beta_{\mathrm{c}} / 7 & -6 \gamma_{\mathrm{c}} / 5 & -24 \beta_{\mathrm{c}} / 35 \\
24 \beta_{\mathrm{c}} / 35 & 12 \gamma_{\mathrm{c}} / 7 & -12+4 \beta_{\mathrm{c}} / 15 & 12 \gamma_{\mathrm{c}} / 7 \\
0 & 20 \beta_{\mathrm{c}} / 21 & 20 \gamma_{\mathrm{c}} / 9 & -20+20 \beta_{\mathrm{c}} / 77
\end{array}\right),} \\
& {\left[\boldsymbol{A}_{1}\right]=\cos \omega^{\prime} t_{1}\left(\begin{array}{cccc}
4 \beta_{\mathrm{c}} / 5 & -2 \gamma_{\mathrm{c}} / 3 & -4 \beta_{\mathrm{c}} / 5 & 0 \\
6 \gamma_{\mathrm{c}} / 5 & 4 \beta_{\mathrm{c}} / 7 & -6 \gamma_{\mathrm{c}} / 5 & -48 \beta_{\mathrm{c}} / 35 \\
48 \beta_{\mathrm{c}} / 35 & 12 \gamma_{\mathrm{c}} / 7 & -8 \beta_{\mathrm{c}} / 15 & 12 \gamma_{\mathrm{c}} / 7 \\
0 & 40 \beta_{\mathrm{c}} / 21 & 20 \gamma_{\mathrm{c}} / 9 & 40 \beta_{\mathrm{c}} / 77
\end{array}\right) \equiv \cos \omega^{\prime} t_{1}\left[\boldsymbol{a}_{1}\right]} \\
& {\left[\boldsymbol{A}_{2}\right]=\cos ^{2} \omega^{\prime} t_{1}\left(\begin{array}{cccc}
2 \beta_{\mathrm{c}} / 5 & 0 & -2 \beta_{\mathrm{c}} / 5 & 0 \\
0 & 2 \beta_{\mathrm{c}} / 7 & 0 & -24 \beta_{\mathrm{c}} / 35 \\
24 \beta_{\mathrm{c}} / 35 & 0 & -4 \beta_{\mathrm{c}} / 15 & 0 \\
0 & 20 \beta_{\mathrm{c}} / 21 & 0 & 20 \beta_{\mathrm{c}} / 77
\end{array}\right) \equiv \cos ^{2} \omega^{\prime} t_{1}\left[\boldsymbol{a}_{2}\right] .}
\end{aligned}
$$


By analogy to Eq. (10), we obtain the vector expansion of $\boldsymbol{F}\left(t_{1}, \varepsilon\right)$ :

$$
\boldsymbol{F}\left(t_{1}, \varepsilon\right)=\boldsymbol{F}_{0}+\varepsilon \boldsymbol{F}_{1}+\varepsilon^{2} \boldsymbol{F}_{2}+\ldots
$$

where:

g) For system (7a)

$$
\begin{aligned}
& \boldsymbol{F}_{0}=\left(\begin{array}{c}
2 \beta_{\mathrm{c}} / 3 \\
2 \beta_{\mathrm{c}} / 5
\end{array}\right), \quad \boldsymbol{F}_{1}=\cos \omega^{\prime} t_{1}\left(\begin{array}{c}
2 \beta_{\mathrm{c}} / 3 \\
4 \beta_{\mathrm{c}} / 5
\end{array}\right) \equiv \cos \omega^{\prime} t_{1} \cdot f_{1}, \\
& \boldsymbol{F}_{2}=\cos ^{2} \omega^{\prime} t_{1}\left(\begin{array}{c}
0 \\
2 \beta_{\mathrm{c}} / 5
\end{array}\right) \equiv \cos ^{2} \omega^{\prime} t_{1} \cdot f_{2} .
\end{aligned}
$$

h) For system (7b)

$$
\begin{aligned}
& \boldsymbol{F}_{0}=\left(\begin{array}{c}
2 \beta_{c} / 3 \\
2 \beta_{c} / 5 \\
0
\end{array}\right), \quad \boldsymbol{F}_{1}=\cos \omega^{\prime} \iota_{1}\left(\begin{array}{c}
2 \beta_{c} / 3 \\
4 \beta_{c} / 5 \\
0
\end{array}\right) \equiv \cos \omega^{\prime} t_{1} \cdot f_{1}, \\
& \boldsymbol{F}_{2}=\cos ^{2} \omega^{\prime} t_{1}\left(\begin{array}{c}
0 \\
2 \beta_{c} / 5 \\
0
\end{array}\right) \equiv \cos ^{2} \omega^{\prime} t_{1} \cdot f_{2} .
\end{aligned}
$$

i) For system (7c)

$$
\begin{aligned}
& \boldsymbol{F}_{0}=\left(\begin{array}{c}
2 \beta_{\mathrm{c}} / 3 \\
2 \beta_{\mathrm{c}} / 5 \\
0 \\
0
\end{array}\right), \quad \boldsymbol{F}_{1}=\cos \omega^{\prime} t_{1}\left(\begin{array}{c}
2 \beta_{\mathrm{c}} / 3 \\
4 \beta_{\mathrm{c}} / 5 \\
0 \\
0
\end{array}\right) \equiv \cos \omega^{\prime} t_{1} \cdot f_{1} \\
& \boldsymbol{F}_{2}=\cos ^{2} \omega^{\prime} t_{1}\left(\begin{array}{c}
0 \\
2 \beta_{\mathrm{c}} / 5 \\
0 \\
0
\end{array}\right) \equiv \cos ^{2} \omega^{\prime} t_{1} \cdot \boldsymbol{f}_{2}
\end{aligned}
$$

Thus, we write the vector $Y\left(t_{1}, \varepsilon\right)$ as

$$
\boldsymbol{Y}\left(t_{1}, \varepsilon\right)=\boldsymbol{Y}_{0}+\varepsilon \boldsymbol{Y}_{1}+\varepsilon^{2} \boldsymbol{Y}_{2}+\ldots
$$

Substituting (10), (11) and (12) into Eq. (9) and equating the coefficients of successive powers of $\varepsilon$, we obtain

$$
\begin{aligned}
& \frac{\mathrm{d} \boldsymbol{Y}_{0}}{\mathrm{~d} t}=\left[A_{0}\right] \boldsymbol{Y}_{0}+\boldsymbol{F}_{0}, \quad \frac{\mathrm{d} \boldsymbol{Y}_{1}}{\mathrm{~d} t}=\left[\boldsymbol{A}_{0}\right] \boldsymbol{Y}_{1}+\left[\boldsymbol{A}_{1}\right] \boldsymbol{Y}_{0}+\boldsymbol{F}_{1} \\
& \frac{\mathrm{d} \boldsymbol{Y}_{2}}{\mathrm{~d} t}=\left[\boldsymbol{A}_{0}\right] \boldsymbol{Y}_{2}+\left[\boldsymbol{A}_{2}\right] \boldsymbol{Y}_{0}+\left[\boldsymbol{A}_{1}\right] \boldsymbol{Y}_{1}+\boldsymbol{F}_{2} \\
& \boldsymbol{Y}_{n}(0, \varepsilon)=0, \quad n=0,1,2 .
\end{aligned}
$$


At any reduced time, the vector $\boldsymbol{Y}_{n}\left(t_{1}\right)$ can be formally written as the superposition of two solutions: the first describing the transient behavior $T_{n}\left(t_{1}\right)$ of the system, and the second - its steady-state evolution $Z_{n}\left(t_{1}\right)$ :

$$
Y_{n}\left(t_{1}\right)=T_{n}\left(t_{1}\right)+Z_{n}\left(t_{1}\right)
$$

For the system (7a), for example, we can obtain the characteristic time $\tau$, at the end of which the transient regime vanishes, by solving the characteristic equation of $\left[A_{0}\right]$, i.e.

$$
\operatorname{det}\left(\left[\boldsymbol{A}_{0}\right]-\lambda \boldsymbol{I}\right)=0 \text {, }
$$

where $\lambda$ is an eigenvalue of $\left[A_{0}\right], I-$ the identity matrix.

Expanding Eq. (15), we obtain for system (7a):

$$
\lambda^{2}+2 \lambda\left(4-\frac{12}{35} \beta_{\mathrm{c}}\right)-2\left(-\frac{2}{35} \beta_{\mathrm{c}}^{2}-\frac{2}{5} \gamma_{\mathrm{c}}^{2}+\frac{52}{35} \beta_{\mathrm{c}}-6\right)=0 .
$$

The discriminant $\Delta^{\prime}$ of Eq. (16) is

$$
\begin{aligned}
& \Delta^{\prime}=4\left\{1+\frac{\gamma_{c}^{2}}{5}\left[\frac{2}{7} \frac{\beta_{c}}{\gamma_{c}^{2}}+\frac{1}{7}\left(\frac{\beta_{c}}{\gamma_{c}}\right)^{2}\left(\frac{36}{35}-1\right)-1\right]\right\}, \\
& \sqrt{\Delta} \approx 2+\frac{2}{35} \frac{\gamma_{c}^{2}}{R}-\frac{\gamma_{c}^{2}}{5}
\end{aligned}
$$

with $R=\gamma_{\mathrm{c}}^{2} / \beta_{\mathrm{c}}\left(\gamma_{\mathrm{c}}\right.$ and $\left.\beta_{\mathrm{c}} \ll 1\right)$. We obtain approximately

$$
\begin{aligned}
& \lambda_{1}=-2+\frac{14}{35} \frac{\gamma_{c}^{2}}{R}-\frac{\gamma_{c}^{2}}{5}=-\frac{1}{\tau_{1}}, \\
& \lambda_{2}=-6+\frac{10}{35} \frac{\gamma_{c}^{2}}{R}+\frac{\gamma_{c}^{2}}{5}=-\frac{1}{\tau_{2}}, \\
& \tau_{1} \approx \frac{1}{2} \quad \text { and } \quad \tau_{2} \approx \frac{1}{6}<\tau_{1} .
\end{aligned}
$$

Thus, after a reduced characteristic time $\tau_{0} \geq \tau_{1}$, the system evolves to a steady regime. We note that the mentioned characteristic time is in excellent agreement with the numerical results of Lce et al. [20] and with the analytical solution of Ilounkonnou [27].

In the following, we are intercsted in the steady-state solutions for all systems. (7). Thus, Eq. (14) is reduced to

$$
Y_{n}\left(t_{1}\right)=Z_{n}\left(t_{1}\right) \text {. }
$$

Therefore, it is straightforward to find

$$
Z_{0}\left(t_{1}\right)=-\left[A_{0}\right]^{-1} F_{0} .
$$

$\boldsymbol{F}_{0}$ is well known. Hence, the vector $\boldsymbol{Z}_{0}$ is well defined. Explicitly, we obtain using Taylor expansion (using REDUCE):

j) for system (7a):

$$
Z_{0}^{2}\left(t_{1}\right)=\left(\begin{array}{c}
\gamma_{c}\left(37 \beta_{c}^{2}+210 \beta_{c}-105 \gamma_{c}^{2}+1575\right) / 4725 \\
\left(5 \beta_{c}^{2}+105 \beta_{c}^{2}+399 \beta_{c} \gamma_{c}^{2}+2205 \beta_{c}+2205 \gamma_{c}^{2}\right) / 33075
\end{array}\right)
$$


k) for system (7b):

$$
Z_{0}^{3}\left(t_{1}\right)=\left(\begin{array}{c}
\gamma_{\mathrm{c}}\left(2 \beta_{\mathrm{c}}^{2}+42 \beta_{\mathrm{c}}-21 \gamma_{\mathrm{c}}^{2}+315\right) / 945 \\
\left(\beta_{\mathrm{c}}^{3}+21 \beta_{\mathrm{c}}^{2}+42 \beta_{\mathrm{c}} \gamma_{\mathrm{c}}^{2}+441 \beta_{\mathrm{c}}+441 \gamma_{\mathrm{c}}^{2}\right) / 6615
\end{array}\right)
$$

l) for system $(7 \mathrm{c})$

$$
Z_{0}^{1}\left(t_{1}\right)=\left(\begin{array}{c}
\gamma_{c}\left(2 \beta_{c}^{2}+42 \beta_{c}-21 \gamma_{c}^{2}+315\right) / 945 \\
\left(\beta_{c}^{3}-15 \beta_{c}^{2}-30 \beta_{c} \gamma_{c}^{2}-315 \beta_{c}-315 \gamma_{c}^{2}\right) / 4725
\end{array}\right),
$$

where $Z_{0}^{i}\left(t_{1}\right)$ is the vector of components $y_{1}\left(l_{1}\right)$ and $y_{2}\left(t_{1}\right)$ defining the nonlinear electric polarization and birefringence to zeroth order of $\varepsilon$. II ere, the superscript $i$ indicates the number of set of differential equations taken account of in solving Eqs. (18).

The best way to appreciate the truncature effect is to evaluate the difference vector of these solutions. This operation gives, defining by three vectors $\left(Z_{0}^{4}\left(t_{1}\right)-\right.$ $\left.Z_{0}^{3}\left(t_{1}\right)\right),\left(Z_{0}^{3}\left(t_{1}\right)-Z_{0}^{2}\left(t_{1}\right)\right),\left(Z_{0}^{4}\left(l_{1}\right)-Z_{0}^{2}\left(l_{1}\right)\right)$, arranged as the columns of a $2 \times 3$ matrix $\Delta Z$ :

$$
\Delta Z=\left(\begin{array}{ccc}
0 & -\beta_{\mathrm{c}}^{2} \gamma_{\mathrm{c}} / 175 & -\beta_{\mathrm{c}}^{2} \gamma_{\mathrm{c}} / 175 \\
-4 \beta_{\mathrm{c}}^{3} / 11025 & -\beta_{\mathrm{c}} \gamma_{\mathrm{c}}^{2} / 175 & -\beta_{\mathrm{c}}\left(4 \beta_{\mathrm{c}}^{2}+63 \gamma_{\mathrm{c}}^{2}\right) / 11025
\end{array}\right) .
$$

In the case of very small molccular rcorientation energy related to the agitation thermal energy $k_{\mathrm{B}} T, \beta_{\mathrm{c}}$ and $\gamma_{\mathrm{c}} \ll 1, \Delta Z$ reduces to a zero matrix 0 . Therefore, $Z_{0}^{2}\left(t_{1}\right)$ is a good approximation to describe nonlinear Kerr effect relaxations.

In the following, we will consider only the system (7a). For simplification, we will denote all solutions by $Z_{j}, j$ being the order of perturbation.

To the first order of $\varepsilon$, we have

$$
\frac{\mathrm{d} Z_{1}}{\mathrm{~d} t}=\left[A_{0}\right] Z_{1}+\left[A_{1}\right] Z_{0}+F_{1}=\left[A_{0}\right] Z_{1}+\left(\cos \omega^{\prime} t_{1}\right) \Psi
$$

where

$$
\Psi=-\left[a_{1}\right]\left[A_{0}\right]^{-1} F_{0}+f_{1} .
$$

A particular solution can be written $(\mathrm{d} \Psi / \mathrm{d} l=0)$ :

$$
\boldsymbol{\varphi} \cos \left(\omega^{\prime} \iota_{1}+\delta\right)
$$

where $\delta$ represents a certain phase angle. Exploiting Eqs. (19) and clementary trigonometric relations, we obtain

$$
\begin{aligned}
& -\omega^{\prime} \cos \delta \cdot \varphi=-\left[\boldsymbol{A}_{0}\right] \varphi \sin \delta \\
& -\omega^{\prime} \sin \delta \cdot \varphi=-\left[\boldsymbol{A}_{0}\right] \varphi \cos \delta+\boldsymbol{\Psi}
\end{aligned}
$$

Equation (20a) leads to

$$
\left[\boldsymbol{A}_{0}\right] \varphi=\omega^{\prime} \cot \delta \cdot \varphi \text {. }
$$

Equation (20c) shows that $\varphi$ is an eigenvector of $\left[A_{0}\right]$ corresponding to the eigenvalue $\lambda\left(\lambda=\lambda_{1}, \lambda=\lambda_{2}\right)$ as follows:

$$
\lambda=\omega^{\prime} \cot \delta .
$$

The value $\delta\left(\delta=\delta_{1}, \delta=\delta_{2}\right)$ is thus determined, since $\lambda$ is well known. In addition, the vector $\boldsymbol{\Psi}$ is defined by Eq. (19b). Therefore, the norms of the eigenvectors

$$
\varphi=\varphi_{1},\left(\lambda=\lambda_{1}\right) \text { and } \varphi=\varphi_{2},\left(\lambda=\lambda_{2}\right)
$$


are determined by Eqs. (20b) and (20c) and the general solution $Z_{1}\left(t_{1}\right)$ is entirely defined by the superposition of the particular solutions as follows:

$$
Z_{1}\left(t_{1}\right)=\varphi_{1} \cos \left(\omega^{\prime} \iota_{1}+\delta_{1}\right)+\varphi_{2} \cos \left(\omega^{\prime} \iota_{1}+\delta_{2}\right) .
$$

This vector solution only depends on ac field harmonics and eigenvectors expressed in terms of molecular parameters.

We now examine the solution to the second order of $\varepsilon$. We have

$$
\begin{aligned}
\frac{\mathrm{d} Z_{2}}{\mathrm{~d} t} & =\left[\boldsymbol{A}_{0}\right] Z_{2}+\left[\boldsymbol{A}_{2}\right] Z_{0}+\left[\boldsymbol{A}_{1}\right] Z_{1}+\boldsymbol{F}_{2}-\left[\boldsymbol{A}_{0}\right] \boldsymbol{Z}_{2}-\left[\boldsymbol{A}_{2}\right]\left[\boldsymbol{A}_{0}\right]^{-1} \boldsymbol{F}_{0} \\
& +\boldsymbol{F}_{2}+\cos \left(\omega^{\prime} t_{1}+\delta_{1}\right)\left[\boldsymbol{A}_{1}\right] \boldsymbol{\varphi}_{1}+\cos \left(\omega^{\prime} t_{1}+\delta_{2}\right)\left[\boldsymbol{A}_{1}\right] \boldsymbol{\varphi}_{2} .
\end{aligned}
$$

We can also write as steady oscillating and constant solutions

$$
\begin{gathered}
\boldsymbol{Z}_{2}=\phi \cos \left(2 \omega^{\prime} \iota_{1}+\Delta\right)+\boldsymbol{X}, \quad\left(\frac{\mathrm{d} \boldsymbol{X}}{\mathrm{d} t}=0\right) ; \\
\boldsymbol{X}=\frac{\left[\boldsymbol{A}_{0}\right]^{-1}}{2}\left\{\left[\boldsymbol{a}_{2}\right]\left[\boldsymbol{A}_{0}\right]^{-1} \boldsymbol{F}_{0}-f_{2}-\right\} \\
-\frac{\left[\boldsymbol{A}_{0}\right]^{-1}}{2}\left\{\cos \delta_{1}\left[\boldsymbol{a}_{1}\right] \varphi_{1}+\cos \delta_{2}\left[\boldsymbol{a}_{1}\right] \boldsymbol{\varphi}_{2}\right\} .
\end{gathered}
$$

Analogously to the case of the first-order calculation, exploiting Eqs. (21) and elementary trigonometric transformations, we define $Z_{2}$ by the following relations:

$$
-\phi 2 \omega^{\prime} \sin \Delta=\left[A_{0}\right] \phi \cos \Delta+\frac{1}{2}\left\{\cos \delta_{1}\left[a_{1}\right] \varphi_{1}+\cos \delta_{2}\left[a_{1}\right] \varphi_{2}+\varphi_{3}\right\},
$$

where

$$
\begin{aligned}
& \varphi_{3}=-\left\{\left[a_{2}\right]\left[\boldsymbol{A}_{0}\right]^{-1} \boldsymbol{F}_{0}-f_{2}\right\}, \\
& -\phi 2 \omega^{\prime} \cos \Delta=-\left[\boldsymbol{A}_{0}\right] \phi \sin \Delta-\frac{1}{2}\left\{\sin \delta_{1}\left[a_{1}\right] \varphi_{1}+\sin \delta_{2}\left[a_{1}\right] \varphi_{2}\right\} .
\end{aligned}
$$

Let us put $\phi=\phi_{1}+\phi_{2}+\phi_{3}$, where $\phi_{1}$ is a solution of Eqs. (22a) and (22b), $\phi_{2}=\phi_{2}=0$ and by analogy in a circular way for $\phi_{2}$ and $\phi_{3}$. We obtain

$$
\left(\frac{\cos \Delta_{j}^{i}}{\cos \delta_{i}}-\frac{\sin \Delta_{j}^{i}}{\sin \delta_{i}}\right)\left[A_{0}\right] \phi_{i}=-2 \omega^{\prime}\left(\frac{\sin \Delta_{j}^{i}}{\cos \delta_{i}}+\frac{\cos \Delta_{j}^{i}}{\sin \delta_{i}}\right) \phi_{i}
$$

and

$$
\left[\boldsymbol{A}_{0}\right] \phi_{3}=2 \omega^{\prime} \cot \Delta_{j}^{3} \phi_{3} \quad(i=1,2 ; j=1,2) .
$$

Equations (22c) and (22d) show again that $\phi_{i}$ (normalized by Eqs. (22a) and $(22 b))$ is an eigenvector of $\left[\boldsymbol{A}_{0}\right]$ which corresponds to eigenvalue $\lambda_{j}$ :

$$
\begin{aligned}
& \lambda_{j}=-\frac{\cos \left(\Delta_{j}^{i}-\delta_{i}\right)}{\sin \left(\Delta_{j}^{i}+\delta_{i}\right)} \quad(j=1,2 ; i=1,2), \\
& \lambda_{j}=2 \omega^{\prime} \cot \Delta_{j}^{3} \quad(j=1,2) .
\end{aligned}
$$


Thus, the vector $Z_{2}$ is entirely defined by the superposition of solutions: constant $\boldsymbol{X}$ and $\phi_{i}(i=1,2,3)$ for each eigenvalue $\lambda_{j}$ of $\left[\boldsymbol{A}_{0}\right]$ and therefore to second-order perturbation, the steady solution of the vector Eq. (9) is given by

$$
Z\left(t_{1}\right)=Z_{0}\left(t_{1}\right)+\varepsilon Z_{1}\left(l_{1}\right)+\varepsilon^{2} Z_{2}\left(l_{1}\right) \text {. }
$$

Thus, the second-order steady-state perturbation solutions of electric polarization and birefringence are expressed as functions of single and double ac field harmonics and molecular parameters.

\section{The Sack formalism}

Starting from the Smoluchowski equation of rotational Brownian motion as modified by Sack for the angular distribution function $f(\theta, t)[22,30-32]$ and using the same standard development based on the mean value of the $n$-th Legendre polynomial $[1,3-6,16,17]$, we obtain a set of equations in which the two first Legendre polynomials are directly related and proportional to the electric polarization and birefringence, respectively [22,30-32]. Using the above-mentioned perturbation formalism, we obtain

$$
\begin{aligned}
& {[J] \frac{\mathrm{d}^{2} \boldsymbol{Y}\left(t_{1}, \varepsilon\right)}{\mathrm{d} t^{2}}+\frac{\mathrm{d} \boldsymbol{Y}\left(t_{1}, \varepsilon\right)}{\mathrm{d} t}=\left[\boldsymbol{A}\left(t_{1}, \varepsilon\right)\right] \boldsymbol{Y}\left(t_{1}, \varepsilon\right)+\boldsymbol{F}\left(t_{1}, \varepsilon\right),} \\
& \boldsymbol{Y}(0, \varepsilon)=\frac{\mathrm{d} \boldsymbol{Y}(0, \varepsilon)}{\mathrm{d} t}=\mathbf{0},
\end{aligned}
$$

where all the quantities keep the values expressed in Sec. 3, point (a) and

$$
\begin{aligned}
& \boldsymbol{Y}=\left(\begin{array}{l}
y_{1} \\
y_{2}
\end{array}\right) ; \quad \frac{\mathrm{d} \boldsymbol{Y}\left(\ell_{1}, \varepsilon\right)}{\mathrm{d} t}=\left(\begin{array}{l}
\mathrm{d} y_{1} / \mathrm{d} \ell \\
\mathrm{d} y_{2} / \mathrm{d} \ell
\end{array}\right) ; \quad \frac{\mathrm{d}^{2} \boldsymbol{Y}\left(t_{1}, \varepsilon\right)}{\mathrm{d} t^{2}}=\left(\begin{array}{l}
\mathrm{d}^{2} y_{1} / \mathrm{d} \ell^{2} \\
\mathrm{~d}^{2} y_{2} / \mathrm{d} t^{2}
\end{array}\right), \\
& {[J]=\frac{I D^{2}}{k T}[\boldsymbol{I}]=\alpha[\boldsymbol{I}] .}
\end{aligned}
$$

$I$ is the moment of the inertia about the transverse axis of the molecule.

The perturbation expansion leads to

$$
\begin{aligned}
& {[J] \frac{\mathrm{d}^{2} \boldsymbol{Y}_{0}}{\mathrm{~d} t^{2}}+\frac{\mathrm{d} \boldsymbol{Y}_{0}}{\mathrm{~d} t}=\left[\boldsymbol{A}_{0}\right] \boldsymbol{Y}_{0}+\boldsymbol{F}_{0},} \\
& {[J] \frac{\mathrm{d}^{2} \boldsymbol{Y}_{1}}{\mathrm{~d} t^{2}}+\frac{\mathrm{d} \boldsymbol{Y}_{1}}{\mathrm{~d} t}=\left[\boldsymbol{A}_{0}\right] \boldsymbol{Y}_{1}+\left[\boldsymbol{A}_{1}\right] \boldsymbol{Y}_{0}+\boldsymbol{F}_{1},} \\
& {[J] \frac{\mathrm{d}^{2} \boldsymbol{Y}_{2}}{\mathrm{~d} t^{2}}+\frac{\mathrm{d} \boldsymbol{Y}_{2}}{\mathrm{~d} t}=\left[\boldsymbol{A}_{0}\right] \boldsymbol{Y}_{2}+\left[\boldsymbol{A}_{1}\right] \boldsymbol{Y}_{1}+\left[\boldsymbol{A}_{2}\right] \boldsymbol{Y}_{0}+\boldsymbol{F}_{2},} \\
& {[J] \frac{\mathrm{d}^{2} \boldsymbol{Y}_{n}}{\mathrm{~d} l^{2}}+\frac{\mathrm{d} \boldsymbol{Y}_{n}}{\mathrm{~d} t}=\left[\boldsymbol{A}_{0}\right] \boldsymbol{Y}_{n}+\left[\boldsymbol{A}_{1}\right] \boldsymbol{Y}_{n-1}+\left[\boldsymbol{A}_{2}\right] \boldsymbol{Y}_{n-2} \quad(n>2),} \\
& \boldsymbol{Y}_{n}(0, \varepsilon)=\frac{\mathrm{d} \boldsymbol{Y}_{n}(0, \varepsilon)}{\mathrm{d} t}=0, \quad n=0,1,2 \ldots
\end{aligned}
$$

We can determine the reduced characteristic time $\tau$, at the end of which the transient regime vanishes, by solving the homogeneous part of Eq. (23) including only the dominant eigenvalues of $\left[\boldsymbol{A}_{0}\right]$. This operation gives $\tau=0.5$. 
In the following, we are interested in the steady-state solutions of Eq. (23) and denote it $Z_{n}\left(t_{1}\right)$ where $n$ corresponds to the order of perturbation. To the zeroth order of perturbation (Eq. (24a)), it is straightforward to find

$$
\boldsymbol{Z}_{0}\left(t_{1}\right)=-\left[\boldsymbol{A}_{0}\right]^{-1} \boldsymbol{F}_{0}
$$

$\boldsymbol{F}_{0}$ is well known. Hence, the vector $\boldsymbol{Z}_{0}$ is entirely defined by Eq. (25). To the first order of perturbation, substituting a particular solution,

$$
Z_{1}=\varphi \cos \left(\omega^{\prime} t_{1}+\delta\right)
$$

where $\delta$ represents a certain phase angle, Eq. (24b) leads to

$$
\begin{aligned}
& \omega^{\prime 2}[J] \varphi \sin \delta-\omega^{\prime} \cos \delta \cdot \varphi=-\left[\boldsymbol{A}_{0}\right] \varphi \sin \delta, \\
& \omega^{\prime 2}[J] \varphi \cos \delta-\omega^{\prime} \sin \delta \cdot \varphi=-\left[\boldsymbol{A}_{0}\right] \varphi \cos \delta+\Psi
\end{aligned}
$$

with

$$
\Psi=-\left[a_{1}\right]\left[A_{0}\right]^{-1} F_{0}+f_{1} .
$$

From Eq. (26a) we obtain

$$
\left(\left[A_{0}\right]+\omega^{\prime 2}[J]\right) \varphi=\omega^{\prime} \cot \delta \cdot \varphi,
$$

which shows that $\varphi$ is an eigenvector of the master matrix $\left(\left[A_{0}\right]+\omega^{2}[J]\right)$ corresponding to its eigenvalue $\sigma\left(\sigma=\sigma_{1}, \sigma=\sigma_{2}\right)$ as follows:

$$
\sigma=\omega^{\prime} \cot \delta \text {. }
$$

The norms of these eigenvectors are determined by Eqs. (26b) and (26c) and the general solution to the first order of perturbation is entirely defined by the superposition of particular solutions as follows:

$$
Z_{1}\left(t_{1}\right)=\sum_{i=1}^{2} \varphi_{i} \cos \left(\omega^{\prime} \iota_{1}+\delta_{i}\right) .
$$

To the second order of $\varepsilon$, writing a particular solution as

$$
Z_{2}=\phi \cos \left(2 \omega^{\prime} t_{1}+\Delta\right)+\boldsymbol{X} \quad\left(\frac{\mathrm{d} \boldsymbol{X}}{\mathrm{d} t}=0\right)
$$

where

$$
\begin{aligned}
& \boldsymbol{X}=-\frac{\left[\boldsymbol{A}_{0}\right]^{-1}}{2}\left\{\sum_{i=1}^{2} \cos \delta_{i}\left[a_{1}\right] \cdot \varphi_{i}+\varphi_{3}\right\}, \\
& \boldsymbol{\varphi}_{3}=f_{2}-\left[\boldsymbol{a}_{2}\right]\left[\boldsymbol{A}_{0}\right]^{-1} \boldsymbol{F}_{0}
\end{aligned}
$$

$\Delta$ is a certain phase argle.

The use of elementary trigonometric transformations in Eq. (24c) leads to

$$
\begin{aligned}
& \left(\left[\boldsymbol{A}_{0}\right]+4 \omega^{\prime 2}[J]\right) \phi=-2 \omega^{\prime} \tan \Delta \cdot \phi \\
& -\frac{1}{2 \cos \Delta}\left\{\sum_{i=1}^{2} \cos \delta_{i}\left[a_{1}\right] \cdot \varphi_{i}+\varphi_{3}\right\}, \\
& \left(\left[A_{0}\right]+4 \omega^{\prime 2}[J]\right) \phi=2 \omega^{\prime} \cot \Delta \cdot \phi-\frac{1}{2 \sin \Delta}\left\{\sum_{i=1}^{2} \sin \delta_{i}\left[a_{1}\right] \cdot \varphi_{i}+\varphi_{3}\right\} .
\end{aligned}
$$


Let us put $\phi=\sum_{i=1}^{3} \phi_{i}$, where $\phi_{i}$ is a solution of Eqs. (27c) and (27d) when $\phi_{j}=0, j=i$ and by analogy in circular way for each $\phi_{i}$. We obtain

$$
\begin{aligned}
& \left(\frac{\cos \Delta_{j}^{i}}{\cos \delta_{i}}-\frac{\sin \Delta_{j}^{i}}{\sin \delta_{i}}\right)\left(\left[A_{0}\right]+4 \omega^{\prime 2}[J]\right) \phi_{i}=-2 \omega^{\prime}\left(\frac{\sin \Delta_{j}^{i}}{\cos \delta_{i}}+\frac{\cos \Delta_{j}^{i}}{\sin \delta_{i}}\right) \phi_{i}, \\
& \left(\left[A_{0}\right]+4 \omega^{\prime 2}[J]\right) \phi_{3}=2 \omega^{\prime} \cot \Delta_{j}^{3} \cdot \phi_{3} \quad(i=1,2 ; j=1,2) .
\end{aligned}
$$

Equations (28) show again that $\phi_{i}$ (normalized by Eqs. (27)) is an eigenvector of $\left(\left[A_{0}\right]+4 \omega^{\prime 2}[J]\right)$ which corresponds to eigenvalue $\sigma_{j}$ :

$$
\begin{aligned}
& \sigma_{j}=-2 \omega^{\prime} \frac{\cos \left(\Delta_{j}^{i}-\delta_{i}\right)}{\sin \left(\Delta_{j}^{i}+\delta_{i}\right)} \quad(j=1,2 ; i=1,2), \\
& \sigma_{j}=2 \omega^{\prime} \cot \Delta_{j}^{3} \quad(j=1,2) .
\end{aligned}
$$

Thus, the vector $Z_{2}$ is entirely defined by the superposition of solutions: constant $\boldsymbol{X}$ and $\phi_{i}(i=1,2,3)$ for each eigenvalue $\sigma_{j}$ of $\left(\left[\boldsymbol{A}_{0}\right]+4 \omega^{2}[J]\right)$ and therefore to the second-order pcrturbation, the steady-state solution of the vector Eq. (23) is given by

$$
Z\left(t_{1}\right)=Z_{0}\left(t_{1}\right)+\varepsilon Z_{1}\left(l_{1}\right)+\varepsilon^{2} Z_{2}\left(l_{1}\right)
$$

The results obtained agree essentially with those of our previous analysis on the nonlinear Kerr effect [17]: the incrial effects generate an additional contribution within the expression of the master matrix characterizing the nonlinear electric polarization and birefringence. This new term is proportional to the square of the frequency multiplied by the order of perturbation. To the zeroth-order perturbation, this term is equal to zero; to the first order its value is $\omega^{2}[J]$ and to the second order of perturbation, the ineria matrix takes the value $4 \omega^{\prime 2}[J]\left(2 \omega^{\prime}\right.$ corresponding to the frequency of the second order of perturbation).

It is easy to obtain the solution of Eq. (24d) to any order in $\varepsilon$ laking into account, as shown in Eq. (23), that $[\boldsymbol{A}]$ and $[\boldsymbol{F}]$ contain at most quadratic terms in $\varepsilon$. Iteratively, we see immediately that the solution to order $n$ in $\varepsilon$ contains only frequencies up to $n \omega^{\prime}$.

For small inertial coefficient $\alpha$, it is also possible to expand the eigenvectors and eigenvalues of $\left(\left[\boldsymbol{A}_{0}\right]+\omega^{\prime 2}[J]\right)$ in terms of $\left[\boldsymbol{A}_{0}\right]$ (given in a previous work [29]) and to compare solutions with and without inertia, keeping the same physical constants $\Delta \alpha, \mu, \omega$.

With the inertial eflects neglected, our results reduce to those of the well-known relaxation theory of the Kerr effect.

The conditions for which inertial eflects have to be taken into consideration in relaxation phenomena were discussed by Gross [33]. Sack [32] proposed the modified Smoluchowski equation of rotational Brownian motion of molecules in liquids, and determined the conditions of its validity. This equation, which holds for small inertial effects, was applied by Cofley [34] to calculate the orientational autocorrelation functions of spherically symmetric bodies with a moment inertia and a permanent dipole moment, and [35] to study the influence of dipole-dipole coupling on dielectric and Kerr relaxation. The same equation is also successfully 
used recently by Alexiewicz $[36,37]$ to treat the molecular Kerr relaxation theory for liquids in reorienting pulse fields and to take into account the small inertial effects in the time transients of nonlinear electric polarization in liquids. For practical purposes [35] the inertial effects will only start to come into prominence when Brownian movement is used to model high-frequency relaxation processes such as dielectric relaxation and Kerr-effect relaxation.

In the general case, taking into account inertial effects would involve the use of an angular velocity-dependent statistical molecular orientation distribution function; this, in turn, would require the solving of the generalized Liouville equation or Kramers (Fokker-Planck-Kramers) equation for the rotational Brownian motions of the molecules in the liquid [38]. An account of the modern techniques of solving the Kramers equations is to be found in the monograph [39]. Some of these techniques are recently used by Coffey [40] and Déjardin [41]. Nonetheless, the complete analytical solution of the problem is still lacking.

We now analyse the mathematical difficulties arising from these techniques in order to propose [42] an exact analytical solution for the Kerr effect relaxations.

\section{Conclusions}

In the present state of the theoretical development of the Kerr relaxation processes in coupled ac and de fields, we were unable to find in the literature exact results concerning the electric birefringence taking into account nonlinear effects.

Indeed, the system of equations giving the electric polarization and birefringence are very difficult to solve analytically when both ac and dc perturbations are present. Some authors proposed approximate solutions in the linear regimes $[6-8,11,21]$. However, to solve these complex systems account must be taken of the nonlinear effects of specics in a given physical medium. Numerical methods have been used in recent works $[20,22,26]$ to solve this problem. Approximate analytical solutions to this problem were also obtained by means of tedious Laplace transforms [21, 28, 29], but the resulting expressions are not simple to exploit.

The main purpose of this paper was to find another approach to solve this problem: the steady-state nonlinear electric polarization and birefringence in coupled ac and dc fields are expressed explicitly in terms of molecular parameters and ac field harmonics. To take this into account, we applied a second-order perturbation theory which turns out to be sufficient to produce the fundamental harmonics of KEB when the fluid under investigation is perturbed by an alternating field $[4-8,11,12,22,26,29]$. This perturbation theory is very general and we extended its solution to any desired order in $\varepsilon$. In particular, we proved that the effects of higher harmonics in these fields (but less relevant experimentally [8]) are easily obtained by a simple recurrence procedure, even when the inertial effects are included. Prior to any calculations, we justified the degree of truncature, in the infinite set of differential equations, necessary to obtain the essential molecular informations. 
Although all these results are purely theoretical, they provide a basic formalism for the interpretation of experiments on the nonlinear dynamic molecular relaxation processes in coupled ac and dc fields.

Using the experimental values of $\mu$ and $\Delta \alpha$, and choosing the appropriate ac and dc fields, the curves of electric polarization and birefringence can be drawn and compared to the steady experimental data. Conversely, the molecular parameters $\mu$ and $\Delta \alpha$ could be easily extracted from the steady solutions obtained here.

However, the inexistence of experimental data of steady-state time evolution of nonlinear KEB in ac and dc superimposed fields does not permit such comparisons today.

Analysing the results obtained, we also notice the appearance of a single frequency for the first-order solution, whereas the second-order solution leads to double frequency as the fundamental harmonics of KEB. This agrees well with the numerical calculations $[22,26]$.

These general steady-state theoretical expressions provide the basis for discussion of the steady-state harmonic variations in the phenomena of electric polarization and birefringence.

\section{Aknowledgement}

M.N. Hounkonnou is grateful to the Van Buuren Foundation for financial support.

\section{References}

[1] P. Langevin, Radium 7, 249 (1910).

[2] M. Born, Ann. Phys. 55, 77 (1918).

[3] M.J. Pauthenier, J. Phys. Rad. 2, 253 (1921).

[4] S. Brunet, Ph.D. Thesis, University of Montpellier, (France) 1971.

[5] R. Marrony, Ph.D. Thesis, University of Montpellier, (France) 1972.

[6] J.-L. Déjardin, Ph.D. Thesis, University of Montpellier, (France) 1971.

[7] C. Delseny, Ph.D. Thesis, University of Perpignan, (France) 1980.

[8] S. Benet, Ph.D. Thesis, University of Perpignan, (France) 1987.

[9] J.C. Filippini, Ph.D. Thesis, University of Grenoble, (France) 1972.

[10] W.T. Colfey, B.V. Paranjape, Proc. R. Ir. Acad. A 78, 17 (1978).

[11] A. Morita, J. Phys. D 11, 1357 (1978).

[12] H. Watanabe, A. Morita, Adv. Chem. Phys. 56, 255 (1984).

[13] W. Alexiewicz, Mol. Phys. 59, 637 (1986).

[14] A. Peterlin, H.A. Stuart, Z. Phys. 112, 129 (1939).

[15] S. Ogawa, S. Oka, J. Phys. Soc. Jpn. 15, 658 (1960).

[16] B. Thurston, D.L. Bowling, J. Colloid Interface Sci. 30, 34 (1969).

[17] M.N. Hounkonnou, A. Ronveaux, R.P. Hazoume, Physica A 176, 569 (1991).

[18] A.D. Buckingham, J.A. Pople, Proc. Phys. Soc. A 68, 905 (1955). 
[19] H. Benoît, Ann. Phys. (France) 6, 561 (1951).

[20] Y.H. Lee, D. Kim, S.H. Lee, J. Chem. Phys. 91, 5628 (1989).

[21] J.-L. Déjardin, G. Débiais, Physica A 161, 182 (1990).

[22] N.M. Hounkonnou, Ph.D. Thesis, DEA, University of Perpignan, (France) 1989.

[23] H. Watanabe, A. Morita, J. Phys. D 13, L153 (1980).

[24] G. Schwarz, Z. Phys. 145, 563 (1956).

[25] C.T. O'Konski, S. Krause, J. Phys. Chem. 74, 3243 (1970).

[26] M.N. Hounkonnou, J. Chem. Soc. Faraday Trans. 87, 297 (1991).

[27] M.N. Hounkonnou, unpublished.

[28] A. Morita, Phys. Rev. A 34, 1499 (1986).

[29] A. Morita, H. Watanabe, Phys. Rev. A 35, 2690 (1987).

[30] W. Alexiewicz, Acta Phys. Pol. A72, 753 (1987).

[31] R.A. Sack, Proc. Phys. Soc. B 70, 402 (1957).

[32] R.A. Sack, Physica 22, 917 (1956).

[33] E.P. Gross, J. Chem. Phys. 23, 1415 (1955).

[34] W.T. Cofley, J. Phys. D 10, 283 (1977); Chem. Phys. Lett. 52, 394 (1977); Chem. Phys. Lett. 54, 519 (1978).

[35] W.T. Coffey, Nonlinear Behaviour of Molecules, Atoms and Ions in Electric Magnelic or Electromagnetic Fields, Elsevier, 1985, p. 411.

[36] W. Alexiewicz, Mol. Phys. 64, 81 (1988).

[37] W. Alexiewicz, Acta Phys. Pol. A6, 753 (1987).

[38] S. Chandrasekhar, Rev. Mod. Phys. 15, 1 (1943).

[39] H. Risken, The Fokker-Planck Equation Methods of Solution and Applications, Springer-Verlag, Berlin 1984.

[40] W.T. Coffey, J. Chem. Phys. 93, 724 (1990); Chem. Phys. 143, 171 (1990); J. Chem. Phys. 95, 2026 (1991).

[41] J.-L. Déjardin, J. Chem. Phys. 95, 576 (1991).

[42] M.N. Hounkonnou, A. Ronveaux, to be published. 